\title{
A Note on Absorption Probabilities in One-dimensional Random Walk via Complex-valued Martingales
}

\author{
Dennis Gilliland \\ Shlomo Levental \\ Yimin Xiao* \\ Department of Statistics and Probability \\ Michigan State University
}

September 25, 2006

\begin{abstract}
Let $\left\{X_{n}, n \geq 1\right\}$ be a sequence of i.i.d. random variables taking values in a finite set of integers, and let $S_{n}=S_{n-1}+X_{n}$ for $n \geq 1$ and $S_{0}=0$, be a random walk on $\mathbb{Z}$, the set of integers. By using the zeros, together with their multiplicities, of the rational function $f(x)=\mathbb{E}\left(x^{X}\right)-1, x \in \mathbb{C}$, we characterize the space $U$ of all complex-valued martingales of the form $\left\{g\left(S_{n}\right), n \geq 0\right\}$ for some function $g: \mathbb{Z} \rightarrow \mathbb{C}$. As an application we calculate the absorption probabilities of the random walk $\left\{S_{n}, n \geq 0\right\}$ by applying the optional stopping theorem simultaneously to a basis of the martingale space $U$. The advantage of our method over the classical approach via the Markov chain techniques (cf. Kemeny and Snell (1960)) is in the size of the matrix that is needed to be inverted. It is much smaller by our method. Some examples are presented.
\end{abstract}

Running head: Absorption Probabilities of Random Walks 2000 AMS Classification numbers: 60G40,60G42; 60G50.

Key words: Random walks, martingales, optional stopping theorem, absorption probabilities.

*Research partially supported by NSF grant DMS-0404729. 


\section{Introduction and main results}

We deal here with a random walk $\left\{S_{n}, n \geq 0\right\}$ on $\mathbb{Z}$. Specifically:

$$
S_{n}=S_{n-1}+X_{n} \text { for } n \geq 1 \text { and } S_{0}=0 \text {, }
$$

where $\left\{X, X_{n}, n \geq 1\right\}$ are i.i.d. random variables on some probability space $(\Omega, \mathcal{F}, \mathbb{P})$ taking values in a finite subset of $\mathbb{Z}$. We will consider 3 cases:

Case 1. Two-sided random walk

$$
\begin{array}{ll}
p_{i}=\mathbb{P}(X=i), \quad i=0,1, \ldots, a ; \\
q_{i}=\mathbb{P}(X=-i), \quad i=1,2, \ldots, b,
\end{array}
$$

where $p_{a}>0, q_{b}>0$ and $\sum_{i=0}^{a} p_{i}+\sum_{i=1}^{b} q_{i}=1$.

Case 2. Right-sided random walk

$$
\begin{aligned}
& p_{i}=\mathbb{P}(X=i), \quad i=0,1, \ldots, a, \\
& \sum_{i=0}^{a} p_{i}=1 \quad \text { and } \quad p_{a}>0 .
\end{aligned}
$$

Case 3. Left-sided random walk

$$
\begin{aligned}
& q_{i}=\mathbb{P}(X=-i), \quad i=0,1, \ldots, b, \\
& \sum_{i=0}^{b} q_{i}=1 \quad \text { and } \quad q_{b}>0 .
\end{aligned}
$$

In all the cases, without loss of generality for our purpose, we assume that the integers in $I \equiv\{k \in \mathbb{Z}: \mathbb{P}(X=k)>0\}$ don't have a common divisor larger than 1. (If not, then $S_{n} \in r \cdot \mathbb{Z}$ where $r>1$ is the largest common divisor of $I$ and we will replace $X$ by $X / r$, etc.)

Let $\mathbb{A}$ be the collection of all integers that $\left\{S_{n}, n \geq 0\right\}$ will ever visit with positive probability, i.e.,

$$
\mathbb{A}=\left\{m \in \mathbb{Z}: \sum_{n=0}^{\infty} \mathbb{P}\left(S_{n}=m\right)>0\right\} .
$$

It follows from elementary results in number theory that in Cases 1, 2 and 3, respectively,

$$
\begin{aligned}
& \mathbb{A}=\mathbb{Z}, \\
& \mathbb{Z}^{+} \supseteq \mathbb{A} \supseteq\left\{m \geq m_{0}\right\}, \text { for some } m_{0} \geq 0, \text { and } \\
& \mathbb{Z}^{-} \supseteq \mathbb{A} \supseteq\left\{m \leq m_{1}\right\}, \text { for some } m_{1} \leq 0,
\end{aligned}
$$

where $\mathbb{Z}^{+}=\{0,1,2, \ldots$,$\} and \mathbb{Z}^{-}=\{0,-1,-2, \ldots$,$\} .$

In order to describe our results, let

$$
f(x)=\mathbb{E}\left(x^{X}\right)-1, \quad x \in \mathbb{C} \backslash\{0\},
$$


where $\mathbb{C}$ denotes the field of complex numbers. Corresponding to the three cases, we have

$$
\begin{aligned}
f(x) & =\sum_{i=0}^{a} p_{i} x^{i}-1+\sum_{i=1}^{b} q_{i} x^{-i}, \\
f(x) & =\sum_{i=0}^{a} p_{i} x^{i}-1 \\
f(x) & =\sum_{i=0}^{b} q_{i} x^{-i}-1 .
\end{aligned}
$$

Let $\left\{x_{j}\right.$ with multiplicity $\left.k_{j}, j \in J\right\}$ denote the roots, over $\mathbb{C}$, of $f(x)=0$. It follows that in the three cases, $\sum_{j \in J} k_{j}=a+b, a, b$, respectively.

Our first result characterizes $U$, the space of all functions $g: \mathbb{A} \rightarrow \mathbb{C}$ such that $\left\{g\left(S_{n}\right), n \geq\right.$ $0\}$ is a martingale with respect to the filtration $\left\{\mathcal{F}_{n}, n \geq 0\right\}$, where $\mathcal{F}_{0}=\{\emptyset, \Omega\}$ and $\mathcal{F}_{n} \equiv$ $\sigma\left(X_{1}, \ldots, X_{n}\right)$ for all $n \geq 1$. When there is no confusion, we will also call $U$ the space of all martingales of the form $\left\{g\left(S_{n}\right), n \geq 0\right\}$.

Proposition 1.1 In the three cases, $U$ is a linear space over $\mathbb{C}$ with dimension $a+b, a$ and $b$, respectively. A basis for $U$ is given by

$$
\left\{g^{j, l}(\cdot): l=0,1, \ldots, k_{j}-1 ; j \in J\right\},
$$

where $g^{j, l}(\cdot): \mathbb{A} \rightarrow \mathbb{C}$ is the function defined by $g^{j, l}(m)=\left(x_{j}\right)^{m} \cdot m^{l}, \forall m \in \mathbb{A}$. Here and in the sequel, we use the convention $0^{0}=1$.

For any given integers $c>0$ and $d<0$, we will consider the stopping time

$$
\tau=\inf \left\{n \geq 0: S_{n} \leq d \text { or } S_{n} \geq c\right\} .
$$

Then $\tau$ can be thought of as the time when a gambler stops betting as soon as he wins at least $c$ betting units or loses at least $|d|$ units. The stopping time $\tau$ is also of interest in connection with sequential sampling; see Feller (1968) for further information.

Let $i=1,2,3$ denote the three cases and let

$$
\begin{aligned}
& V_{1} \equiv\{d-b+1, \ldots, d\} \cup\{c, \ldots, c+a-1\}, \\
& V_{2} \equiv\{c, \ldots, c+a-1\}, \\
& V_{3} \equiv\{d-b+1, \ldots, d\} .
\end{aligned}
$$

Then for each $i=1,2,3, \tau<\infty$ if and only if $S_{n} \in V_{i}$ for some $n \geq 1$. We will denote the cardinality of $V_{i}$ by $\# V_{i}$.

Our main task in this paper is to calculate the distribution of $S_{\tau}$ :

$$
\alpha_{k} \equiv \mathbb{P}\left(S_{\tau}=k\right), \quad k \in V_{i},
$$

which will be called the absorption probabilities of the random walk $\left\{S_{n}, n \geq 0\right\}$.

The following is our main result. It is formulated for all the three cases. 
Theorem 1.2 For every $i=1,2,3$, let $\left\{g^{j, l}(\cdot)\right\}_{0 \leq l \leq k_{j}-1, j \in J}$ be the basis of $U$ given in Proposition 1.1. Let

$$
B_{i}=\left[g^{j, l}(k)\right]_{0 \leq l \leq k_{j}-1, j \in J ; k \in V_{i}}
$$

denote the associated $\# V_{i} \times \# V_{i}$ matrix over $\mathbb{C}$, where $(j, l)$ and $k$ are the indices of the rows and columns, respectively. Then $B_{i}$ is non-singular and the column vector $\left(\alpha_{k}\right)_{k \in V_{i}}$ of absorption probabilities is given by

$$
\left(\alpha_{k}\right)_{k \in V_{i}}=B_{i}^{-1} \cdot \mathbf{g}(0),
$$

where $\mathbf{g}(0)$ denotes the $\# V_{i}$-dimensional column vector $\left(g^{j, l}(0)\right)_{0 \leq l \leq k_{j}-1, j \in J}$.

The equation $B_{i} \cdot\left(\alpha_{k}\right)=\mathbf{g}(0)$ is obtained by applying simultaneously the optional stopping theorem to the complex-valued martingales $\left\{g^{j, l}\left(S_{n}\right): n \geq 0\right\}\left(0 \leq l \leq k_{j}-1, j \in J\right)$. The main difficulty in proving Theorem 1.2 is to prove in Case 1 that $B_{1}$ is non-singular. This difficulty is due to the "gap" between the powers that appear in $B_{1}$. It is overcome by using Lemma 3.1, which is a linear algebra type of result that may be of independent interest.

Now we compare our technique with the classical Markov chain approach and the related work of Feller (1968). To apply Theorem 1.2, in the setup of Case 1, we need to invert an $(a+b) \times(a+b)$ matrix. In the Markov chain approach (see, e.g., Kemeny and Snell (1960)) one defines a transition matrix on $(d-b+1, \ldots, c+a-1)$ with $V_{1}$ taken to be the absorbing states and then inverts the matrix $I-Q$, where $I$ is the $(c-d-1) \times(c-d-1)$ identity matrix and $Q$ is the transition matrix restricted to the transient states $(d+1, \ldots, c-1)$. The matrix $I-Q$ is of dimension $(c-d-1) \times(c-d-1)$. Usually the dimension of $B_{1}$ in Theorem 1.2 is much smaller than that of $I-Q$.

Feller (1968, pp. 363-367) deals with the same setup as our Case 1 with a goal of finding $\mathbb{P}\left(S_{\tau} \leq d\right)$ ("ruin probability"). Like us he makes use of the roots of $f(x)=0$. However he doesn't use explicit martingale concepts and, more importantly, he doesn't prove explicitly that $B_{1}$ is non-singular. From the point of view of this paper Feller starts by defining the boundary function $u: V_{1} \rightarrow \mathbb{C}$ by

$$
u(m)= \begin{cases}1 & \text { if } d-b+1 \leq m \leq d \\ 0 & \text { if } c \leq m \leq c+a-1\end{cases}
$$

By the invertibility of $B_{1}$ there is a unique representation:

$$
u(m)=\sum_{j, l} A_{j, l} g^{j, l}(m), \quad m \in V_{1}
$$

for some complex numbers $A_{j, l}\left(0 \leq l \leq k_{j}-1, j \in J\right)$.

By extending the definition of $u$, via that representation for $m \in \mathbb{Z}$ we get that $u \in U$, namely $\left\{u\left(S_{n}\right), n \geq 0\right\}$ is a martingale. That leads by the optional stopping theorem to the formula

$$
\mathbb{P}\left(S_{\tau} \leq d\right)=\sum_{j, l} A_{j, l} g^{j, l}(0)
$$


Feller (1968) also gives upper and lower bounds for $\mathbb{P}\left(S_{\tau} \leq d\right)$ by using, in our context, the 2 martingales associated with the positive roots of $f(x)=0$. The reader is referred to Ethier and Khoshenevisan (2002) for a different approach regarding estimating $\mathbb{P}\left(S_{\tau} \leq d\right)$ more precisely from above and below.

The rest of this paper is organized as follows. In Section 2, we prove Proposition 1.1. The proof of Theorem 1.2 is given in Section 3. In Section 4, we give some further remarks and examples related to Proposition 1.1 and Theorem 1.2.

\section{Martingales of the form $\left\{g\left(S_{n}\right), n \geq 0\right\}$}

Now we prove Proposition 1.1.

Proof of Proposition 1.1 Note that, for any function $g: \mathbb{A} \rightarrow \mathbb{C},\left\{g\left(S_{n}\right): n \geq 0\right\}$ is a martingale (with respect to the filtration $\left\{\mathcal{F}_{n}\right\}$ ) if and only if

$$
\mathbb{E}(g(m+X))=g(m), \quad \forall m \in \mathbb{A} .
$$

The fact that $U$ is a linear space is obvious. In Case 1 its dimension is $a+b$ since $\{g(m), m=$ $-b, \ldots, 0, \ldots, a-1\}$ can be defined arbitrarily and, once they are defined, then $g$ is uniquely defined on all $\mathbb{Z}$. Indeed $p_{a}>0$ and $\mathbb{E}(g(X))=g(0)$ together define $g(a)$ uniquely, and subsequently defines $g$ for all integers larger than $a$. Similarly $q_{b}>0$ and $\mathbb{E}(g(-1+X))=g(-1)$ uniquely define $g(-b-1)$, and subsequently defines $g$ for all integers smaller than $-b$.

In Case 2 we define arbitrarily $\left\{g(m), m=m_{0}, \ldots, m_{0}+a-1\right\}$ and, as in Case 1, we can exploit (2.1) to define $g$ on $\mathbb{A}$. For example if $m$ is the largest integer in $\mathbb{A}$ among those smaller than $m_{0}$ then $g(m)=\mathbb{E}(g(m+X))$ directly defines $g(m)$. The proof of Case 3 is similar to Case 2 , and is omitted.

We next show that $\left\{g^{j, l}\right\} \subseteq U$. Since $x_{j}$ is a zero of $f(x)$ with multiplicity $k_{j}$ we get

$$
f^{(l)}\left(x_{j}\right)=0, \quad \forall l=0,1, \ldots, k_{j}-1 .
$$

A small calculation reveals that (2.2) is equivalent to

$$
\begin{aligned}
& \mathbb{E}\left[\left(x_{j}\right)^{X}\right]=1, \\
& \mathbb{E}\left[\left(x_{j}\right)^{X} X^{l}\right]=0 \quad \text { for every } l=1, \ldots, k_{j}-1 .
\end{aligned}
$$

The proof that $\left\{g^{j, l}\right\}$ satisfies relationship (2.1) follows from (2.3): $\forall m \in \mathbb{A}$,

$$
\begin{aligned}
\mathbb{E}\left[\left(x_{j}\right)^{m+X} \cdot(m+X)^{l}\right] & =\left(x_{j}\right)^{m} \mathbb{E}\left[\left(x_{j}\right)^{X} \cdot \sum_{i=0}^{l}\left(\begin{array}{l}
l \\
i
\end{array}\right) m^{i} X^{l-i}\right] \\
& =\left(x_{j}\right)^{m} m^{l} \mathbb{E}\left[\left(x_{j}\right)^{X}\right]+\sum_{i=0}^{l-1}\left(\begin{array}{l}
l \\
i
\end{array}\right) m^{i} \mathbb{E}\left[\left(x_{j}\right)^{X} X^{l-i}\right] \\
& =\left(x_{j}\right)^{m} m^{l} .
\end{aligned}
$$


It remains to show that $\left\{g^{j, l}\right\}_{0 \leq l \leq k_{j}-1, j \in J}$ are linearly independent. The proofs are similar in all cases so we will concentrate only on Case 1. Linear independence is equivalent to the non-singularity of the $(a+b) \times(a+b)$ matrix $\left[g^{j, l}(m)\right]$, in which $(j, l)$ are the $a+b$ indices of the rows and $-b \leq m \leq a-1$ are the $a+b$ indices of the columns. To prove the later statement, we start by assuming that there exist complex numbers $c_{m}(-b \leq m \leq a-1)$ such that

$$
\sum_{m=-b}^{a-1} c_{m} g^{j, l}(m)=\sum_{m=-b}^{a-1} c_{m}\left(x_{j}\right)^{m} \cdot m^{l}=0, \quad \forall j, l .
$$

Let $h(x)=\sum_{m=-b}^{a-1} c_{m} x^{m}$. Then the condition (2.5) is equivalent to $h^{(l)}\left(x_{j}\right)=0, \forall j, l$ which means that $h$ has, when counted with multiplicity, $a+b$ zeros. Unless $h \equiv 0$ the number of zeros of $h$ is $a+b-1$. This finishes the proof of Proposition 1.1.

Remark 2.1 We comment briefly on the positive roots of $f(x)=0$ in Case 1, since they have played important roles in Feller (1968), Ethier and Khoshnevisan (2002). One obvious positive root of $f(x)=0$ is 1 . According to Descartes' rules of signs the number of positive roots of $f(x)=0$ (counted with multiplicity) is bounded by (and equal in parity to) the number of changes of signs of its coefficients $(=2$ in Case 1$)$. It follows that the following are equivalent:

(a) $\mathbb{E}(X)=0$,

(b) 1 is the only positive root of $f(x)=0$ and it has multiplicity 2 ,

(c) $\left\{S_{n}, n \geq 0\right\}$ is a martingale.

Also the following are equivalent:

(a) $\mathbb{E}(X) \neq 0$,

(b) There is exactly one additional positive root of $f(x)=0$ besides 1 , and both have multiplicity 1.

\section{Application to the absorption probabilities.}

For proving Theorem 1.2, we need the following lemma.

Lemma 3.1 Let $P=\left(p_{k, i}\right)_{0 \leq k, i \leq m}$ denote an $m \times m$ real matrix. If for every $1 \leq k \leq m$, the entries in the $k$-th row satisfy the following condition:

$$
p_{k, i}= \begin{cases}\geq 0 & \text { and is nondecreasing in } i \text { for } i \leq k, \\ \leq 0 & \text { and is nondecreasing in } i \text { for } k<i, \\ >0 & \text { if } k=i .\end{cases}
$$

Then $\operatorname{det}(P)>0$, where $\operatorname{det}(P)$ denotes the determinant of $P$. 
Proof The proof is by induction on $m$. For $m=1$, our lemma holds obviously. Now we assume that $m>1$ and the lemma holds for all $(m-1) \times(m-1)$ matrices satisfying (3.1). Then for any $m \times m$ matrix $P$ that satisfies (3.1), we write

$$
P=\left[\begin{array}{cc}
A & B \\
C & p_{m, m}
\end{array}\right],
$$

where $A, B$ and $C$ are $(m-1) \times(m-1),(m-1) \times 1$ and $1 \times(m-1)$ matrices, respectively. It is known from linear algebra (see, for example, Bapat and Raghavan (1996, pp. 139-140)) that

$$
\operatorname{det}(P)=p_{m, m} \cdot \operatorname{det}\left(A-B\left(p_{m, m}\right)^{-1} C\right) .
$$

Using (3.2) we can finish the proof by showing that the $(m-1) \times(m-1)$ matrix $A-$ $B\left(p_{m, m}\right)^{-1} C$ satisfies the condition (3.1) as well.

Let $0 \leq k \leq m-1$ be fixed. The $k$-th row of $-B\left(p_{m, m}\right)^{-1} C$ has the form

$$
\left(-p_{k, m} \cdot \frac{p_{m, i}}{p_{m, m}}, 0 \leq i \leq m-1\right) .
$$

All its members are non-negative since $p_{k, m} \leq 0$ and $p_{m, i} \geq 0$, and are nondecreasing in $i$.

Now we verify that the $k$-th row of the matrix $A-B\left(p_{m, m}\right)^{-1} C$ satisfies (3.1). Clearly,

$$
p_{k, i}-p_{k, m} \cdot \frac{p_{m, i}}{p_{m, m}} \geq 0 \quad \text { for } i \leq k \text { and is nondecreasing in } i .
$$

On the other hand, if $k<i \leq m-1$, then $0 \leq p_{m, i} \leq p_{m, m}$ and $p_{k, i} \leq p_{k, m} \leq 0, k<i \leq m-1$. It follows that

$$
p_{k, i}-p_{k, m} \cdot \frac{p_{m, i}}{p_{m, m}} \leq 0 \quad \text { for } k<i \leq m-1
$$

and it is also nondecreasing in $i$. Thus we conclude that $A-B\left(p_{m, m}\right)^{-1} C$ does satisfy (3.1). The proof is finished.

Now we proceed to proving Theorem 1.2.

Proof of Theorem 1.2 Since all the \# $V_{i}$ complex-valued martingales $\left\{g^{j, l}\left(S_{n}\right): n \geq 0\right\}$ $\left(j \in J, 0 \leq l \leq k_{j}-1\right)$ are bounded on the time interval $[0, \tau]$, we apply the optional stopping theorem simultaneously to derive, in all three cases,

$$
\begin{aligned}
B_{i} \cdot\left(\alpha_{k}\right)_{k \in V_{i}} & =\left(\mathbb{E}\left(g^{j, l}\left(S_{\tau}\right)\right)\right)_{j \in J, 0 \leq l \leq k_{j}-1} \\
& =\left(g^{j, l}(0)\right)_{j \in J, 0 \leq l \leq k_{j}-1} \\
& :=\mathbf{g}(0),
\end{aligned}
$$

where $B_{i}$ is the matrix in (1.1) and all the vectors are column vectors. Hence, (1.2) will follow from (3.3) once we prove that $B_{i}$ is non-singular for $i=1,2,3$. 
To this end, we first observe that the rank of $B_{i}$ is equal to that of the matrix $\widetilde{B}_{i}=$ $\left[\left(x_{j}\right)^{k+m} \cdot(k+m)^{l}\right]$ for every $m \in \mathbb{Z}$. Indeed a "rank preserving" transformation from $B_{i}$ to $\widetilde{B}_{i}$ can be done by applying

$$
\left(x_{j}\right)^{k+m} \cdot(k+m)^{l}=\left(x_{j}\right)^{m} \cdot\left[\sum_{n=0}^{l}\left(\begin{array}{l}
l \\
n
\end{array}\right) m^{l-n} \cdot\left(\left(x_{j}\right)^{k} k^{n}\right)\right] .
$$

This observation proves the non-singularity of $B_{i}$ in Cases 2 and 3. Indeed in Case 2 we have proven (see the proof of Proposition 1.1) that the matrix

$$
\left[\left(x_{j}\right)^{k} \cdot k^{l}\right]_{0 \leq l \leq k_{j}-1, j \in J ; k=0, \ldots, a-1}
$$

is non-singular so we choose $m=-c$ and we are done. Case 3 is similar.

From now on we deal only with Case 1. By our observation it is enough to work with the $(a+b) \times(a+b)$ matrix $\widetilde{B}_{1}=\left[\left(x_{j}\right)^{k} \cdot k^{l}\right]$ with column indices

$$
k \in K \equiv\{0,1, \ldots, b-1\} \cup\{b+m, b+m+1, \ldots, b+m+a-1\},
$$

where $m=c-d-1>1$.

We start by assuming that there are constant $c_{k} \in \mathbb{C}$ for which

$$
\sum_{k \in K} c_{k}\left(x_{j}\right)^{k} k^{l}=0, \quad \forall j \in J, 0 \leq l \leq k_{j}-1 .
$$

We will show $c_{k}=0$ for every $k \in K$.

Define the polynomial $G(x)=\sum_{k \in K} c_{k} x^{k}$. From (3.4) it follows that the roots of $G(x)=0$ are given by

$$
\left\{x_{j} \text { with multiplicity of at least } k_{j}, j \in J\right\} \text {. }
$$

This implies that $G(x)$ is divisible by the polynomial

$$
F(x)=\frac{x^{b} \cdot f(x)}{x-1}
$$

So we can write

$$
G(x)=F(x) \cdot H(x),
$$

where $H(x)=\sum_{k=0}^{m} d_{k} x^{k}, d_{k} \in \mathbb{C}$ and $m=c-d-1$.

We also have $H(1)=0$, since the multiplicity of the root 1 in $F(x)$ is smaller than that of $G(x)$. Moreover, it is easy to see that in fact

$$
F(x)=\sum_{k=b}^{a+b-1} P_{k} x^{k}-\sum_{k=0}^{b-1} Q_{k} x^{k},
$$


where

$$
\begin{aligned}
& P_{k}=\mathbb{P}(X>k-b)=\sum_{i=k-b+1}^{a} p_{i}, \quad \forall k=b, b+1, \ldots, a+b-1, \\
& Q_{k}=\mathbb{P}(X \leq k-b)=\sum_{i=b-k}^{b} q_{i}, \quad \forall k=0,1, \ldots, b-1 .
\end{aligned}
$$

By observing that in the polynomial $G(x)$ the coefficients of $x^{k}$ for $k=b, \ldots, b+m-1$ are identically 0 and by using $H(1)=0$, we get $m+1$ equations:

$$
\left\{\begin{array}{l}
\sum_{\substack{i=(k-a+1)^{+} \\
m}}^{k} P_{k+b-i} d_{i}-\sum_{i=k+1}^{(k+b) \wedge m} Q_{k+b-i} d_{i}=0, \quad \forall k=0,1, \ldots, m-1, \\
\sum_{i=0}^{m} d_{i}=0
\end{array}\right.
$$

where $x^{+}=\max \{x, 0\}$ and $x \wedge y=\min \{x, y\}$.

Our goal is to show that $d_{0}=d_{1}=\cdots=d_{m}=0$ which will imply via (3.5) that $G \equiv 0$ and our task will be done. In other words we need to prove that the matrix, $P=\left[p_{k, i}\right]_{0 \leq i, k \leq m}$ generated by the coefficients of (3.8) is non-singular. By (3.8), we have

$$
p_{k, i}= \begin{cases}P_{k+b-i} & \text { if }(k+1-a)^{+} \leq i \leq k, \text { and } k<m, \\ -Q_{k+b-i} & \text { if } k+1 \leq i \leq(k+b) \wedge m, \text { and } k<m, \\ 1 & \text { if } k=m\end{cases}
$$

Observing each row of $P$ we see that for each $k$,

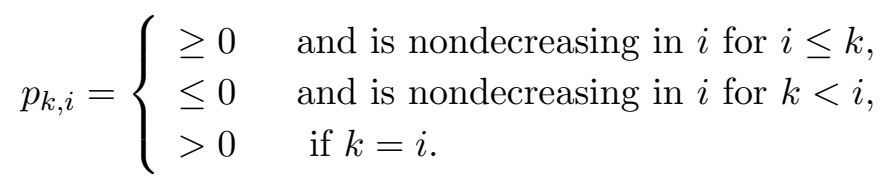

That is, the matrix $P$ satisfies condition (3.1). Hence, the non-singularity of $P$ follows from Lemma 3.1.

\section{Examples}

In this section, we give some examples. Evaluations in Examples $4.2-4.5$ were done with Mathematica5.

Example 4.1 First we consider the special case where $X$ takes 2 values: $\{-b, a\}$, that is, $q_{b}+p_{a}=1$. This setting was considered by Uspensky (1937) and Feller (1968) who obtained upper and lower bounds for their absorption (ruin) probabilities. As Examples 4.2 and 4.3 show, our Theorem 1.2 can be applied to provide exact absorption probabilities.

We now discuss in more detail the properties of the roots of $f(x)=0$. For ease of notation we use $p=p_{a}, q=q_{b}$. To locate roots with multiplicity of at least 2 it will be more convenient to work with the equation $h(x) \equiv p x^{a+b}-x^{b}+q=0$ that is equivalent to $f(x)=0$. 
It follows from Remark 2.1 that $f(x)=0$ has two positive roots, either $x=1$ with multiplicity 2 or two distinct positive roots depending upon whether $\mathbb{E}(X)=0$ or not. Applying Descartes' rules of signs to $h(-x)$, we see that there are no negative roots when both $a$ and $b$ are odd and exactly one negative root when $a$ and $b$ have different parity.

Moreover, since $h^{\prime}(x)=p(a+b) x^{a+b-1}-b x^{b-1}$ we get that

$$
h^{\prime}(x)=0 \Longleftrightarrow x=\left(\frac{b}{(a+b) p}\right)^{1 / a} .
$$

However,

$$
h\left(\left(\frac{b}{(a+b) p}\right)^{1 / a}\right)=0 \Longleftrightarrow p^{b} q^{a}=\left(\frac{b}{a+b}\right)^{b}\left(\frac{a}{a+b}\right)^{a} \Longleftrightarrow p=\frac{b}{a+b},
$$

where the second equivalence can be proved by standard calculus. Since $p=\frac{b}{a+b} \Longleftrightarrow \mathbb{E}(X)=$ 0 , we have shown that if $\mathbb{E}(X) \neq 0$ all the $a+b$ roots are distinct.

If $\mathbb{E}(X)=0$ then by (4.1) we get $h^{\prime}(x)=0 \Rightarrow x^{a}=1$. If in addition $h(x)=0$ we also get: $x^{b}=1$. Since $\{a, b\}$ are relatively prime, $x^{b}=x^{a}=1$ is equivalent to $x=1$. Therefore, $f(x)=0$ has $a+b-1$ distinct complex roots and $x=1$ is a root of multiplicity 2 .

Example 4.2 Here $X$ takes two values $\{-2,1\}$ with probabilities $1 / 3$ and $2 / 3$ so that $\mathbb{E}(X)=0$. For any given integers $d<0, c>0$, the absorbing states are $\{d-1, d, c\}$. The roots of $f(x)=0$ are $1,1, \frac{1}{2}$. The matrix $B_{1}$ and vector $\mathbf{g}(0)$ of Theorem 1.2 are

$$
\left[\begin{array}{llc}
1 & 1 & 1 \\
d-1 & d & c \\
\left(-\frac{1}{2}\right)^{d-1} & \left(-\frac{1}{2}\right)^{d} & \left(-\frac{1}{2}\right)^{c}
\end{array}\right] \quad \text { and } \quad\left[\begin{array}{l}
1 \\
0 \\
1
\end{array}\right]
$$

respectively. Hence the distribution $\left(\alpha_{k}\right)$ of the stopped random walk is given by

\begin{tabular}{|l|l|l|l|}
\hline$k$ & \multicolumn{1}{|c|}{$d-1$} & \multicolumn{1}{c|}{$d$} & \multicolumn{1}{c|}{$c$} \\
\hline$\delta \cdot \alpha_{k}$ & $c\left(1-(-0.5)^{d}\right)-d(1-$ & $(d-1)\left(1-(-0.5)^{c}\right)-$ & $1-d(-0.5)^{d-1}+(d-$ \\
& $\left.(-0.5)^{c}\right)$ & $c\left(1-(-0.5)^{d-1}\right)$ & $1)(-.5)^{d}$ \\
\hline
\end{tabular}

where

$$
\delta=(-0.5)^{c}+(c-d)(-0.5)^{d-1}-(c-d+1)(-0.5)^{d} .
$$

For the special case of $d=-3$ and $c=2$, the absorption probabilities are

\begin{tabular}{|l|c|c|c|}
\hline$k$ & -4 & -3 & 2 \\
\hline$\alpha_{k}$ & $\frac{3}{19}$ & $\frac{4}{19}$ & $\frac{12}{19}$ \\
\hline
\end{tabular}


Example 4.3 A person sent a communication to one of the co-authors in which he proposed (conjectured) a modification of the formula for absorption probabilities for a $\{+1,-1\}$ random walk to handle a $\{+1,-2\}$ random walk. The modification was incorrect but the communication led to the efforts behind this paper. This example is the solution to the specific gambler's ruin problem that was proposed. A gambler has 30 in capital and a goal of 31 . A bet is available to the gambler that results in +1 with probability 0.642 and -2 with probability 0.358 . What is the probability that the gambler gets 31 before the capital has shrunk to 1 or 0 , making the bet unavailable to the gambler? Here $X$ takes two values $\{-2,1\}$ with probabilities 0.358 and 0.642 , respectively, so that $\mathbb{E}(X)=-0.074$. Shifting to start the random walk at 0 leads to the absorbing states $\{-30,-29,1\}$. The roots of $f(x)=1$ are $1, r_{2}=(0.358+\sqrt{1.047508}) / 1.284$ and $r_{3}=(0.358-\sqrt{1.047508}) / 1.284$. The matrix $B_{1}$ and vector $\mathbf{g}(0)$ of Theorem 1.2 are

$$
\left[\begin{array}{lll}
1 & 1 & 1 \\
r_{2}^{-30} & r_{2}^{-29} & r_{2} \\
r_{3}^{-30} & r_{3}^{-29} & r_{3}
\end{array}\right], \quad\left[\begin{array}{l}
1 \\
1 \\
1
\end{array}\right]
$$

and the distribution $\left\{\alpha_{k}\right\}$ of the stopped random walk is given by

\begin{tabular}{|l|c|c|c|}
\hline$k$ & -30 & -29 & 1 \\
\hline$\alpha_{k}$ & 0.027023 & 0.052139 & 0.920838 \\
\hline
\end{tabular}

Example 4.4 Let $X$ be a random variable with distribution given by

\begin{tabular}{|l|c|c|c|c|}
\hline$x$ & -1 & 1 & 2 & 3 \\
\hline$p(x)$ & $\frac{14,401}{24,002}$ & $\frac{5,201}{24,002}$ & $\frac{4,000}{24,002}$ & $\frac{400}{24,002}$ \\
\hline
\end{tabular}

Then $\mathbb{E}(X)=0$ and $f(x)=0$ has a two distinct complex roots. More precisely, the roots of $f(x)=0$ are $x=1$ (multiplicity 2 ) and $x=-6 \pm i / 20$. For any given integers $c>0$ and $d<0$, the absorption probabilities can be computed using Theorem 1.2.

Example 4.5 Finally we present a random variable $X$ such that $f(x)=0$ has a complex root with multiplicity 2. The distribution of $X$ is

\begin{tabular}{|l|c|c|c|c|c|}
\hline$x$ & -5 & -4 & -3 & -2 & 6 \\
\hline$p(x)$ & $\frac{29,093,936}{18,419,419,575}$ & $\frac{487,362,532}{18,419,419,575}$ & $\frac{3,011,713,128}{18,419,419,575}$ & $\frac{7,202,578,104}{18,419,419,575}$ & $\frac{7,688,671,875}{18,419,419,575}$ \\
\hline
\end{tabular}

and $x=\frac{-1}{5}+\frac{i}{15}$ is a root with multiplicity 2. It follows from Proposition 1.1 that both $\left\{\left(\frac{-1}{5}+\frac{i}{15}\right)^{S_{k}}\right\}$ and $\left\{\left(\frac{-1}{5}+\frac{i}{15}\right)^{S_{k}} \cdot S_{k}\right\}$ are martingales. Obviously, $x=\frac{-1}{5}-\frac{i}{15}$ is another root of $f(x)=0$ with multiplicity 2 .

Acknowledgement. We thank Professor Roy Erickson for showing us Example 4.5. 


\section{References}

[1] R. B. Bapat and T. E. S. Raghavan (1996), Nonnegative Matrices and Applications. Cambridge University Press, Cambridge.

[2] S. N. Ethier and D. Khoshenevisan (2002), Bounds on gambler's ruin in terms of moments. Methodology and Computing in Applied Probability 4, 55-68.

[3] W. Feller (1968), An Introduction to Probability Theory and Its Applications, Volume 1, $3^{\text {rd }}$ edition, John Wiley \& Sons, New York.

[4] J. G. Kemeny and J. L. Snell (1960), Finite Markov Chains. Van Nostrand Company.

[5] J. V. Uspensky (1937), Introduction to Mathematical Probability. McGraw-Hill, New York.

Dennis Gilliand. Department of Statistics and Probability, A-413 Wells Hall, Michigan State University, East Lansing, MI 48824, U.S.A.

E-mail: gilliland@stt.msu.edu

Shlomo Levental. Department of Statistics and Probability, A-413 Wells Hall, Michigan State University, East Lansing, MI 48824, U.S.A.

E-mail: levental@stt.msu.edu

Yimin XIAO. Department of Statistics and Probability, A-413 Wells Hall, Michigan State University, East Lansing, MI 48824, U.S.A.

E-mail: xiao@stt.msu.edu

URL: http://www.stt.msu.edu/ ${ }^{\sim}$ xiaoyimi 